\title{
Electromagnetic Radiation towards Adult Human Head from 900MHz Handheld Mobile Phone
}

\author{
D.A.A.Mat ${ }^{1}$, W. T. Franky, K. Kipli, A. Joseph, S. Sahrani, K. Lias, S. Suhaili \\ Department of Electronics Engineering, Faculty of Engineering, Universiti Malaysia Sarawak \\ 94300 Kota Samarahan, Sarawak, Malaysia \\ 'amdazra@feng.unimas.my
}

\begin{abstract}
Electromagnetic radiation produce by mobile phone and the relationship with the human's health is not a new issue nowadays. Since the used of mobile phone had increased rapidly over the past few years, people are becoming more concern with their health when dealing with the so-called electromagnetic radiation. This type of radiation would leads to heating of body tissue at specific rate called the thermal radiation. Thermal radiation depends on the frequency of the energy, the power density of the radio frequency field that strikes the body and the polarization of wave. This paper will discuss on the result collected from the thermal radiation generated by handheld mobile phone with frequency of $900 \mathrm{MHz}$ towards adult human head. The analysis is conducted in a laboratory with average of 45 minutes talking hour with two different types of mobile phone, internal and external antenna. The results show an increased of heat especially at the place near the ear skull after $\mathbf{4 5}$ minutes of operation. When comparing both different types of mobile phone, mobile phone with external antenna produce more heat compared to mobile phone with internal antenna.
\end{abstract}

Keywords - thermal radiation, electromagnetic, mobile phone, human head, internal and external antenna

\section{INTRODUCTION}

Radio frequency exposure produced by mobile phones and the relationship with the human health is a big concern nowadays. Researches and studies had been conducted based on epidemiological and experimental analysis. National Radiological Protection Board (NRPB) or currently known as Health Protection Agency (HPA) has prepared and published a report to provide advice to address public concerns about the mobile technology [1]. Electromagnetic fields (EMF) consists of oscillating electric and magnetic fields which interact differently with biological systems such as cells, plants, animals or human beings. This field is produced by the used of mobile phone especially for longer period of time. A certain amount of energy radiated by handset device due to the presence of EMF is absorbed by the user's body tissue because of the existence of the electromagnetic radiation. Both RF and $60 \mathrm{~Hz}$ fields are classified as non-ionizing radiation due to the low frequency, but still at sufficiently high power densities, the electromagnetic radiation can poses certain health hazards [2]. The high RF energy also can cause injuries by heating body tissue called the thermal effects depend upon the frequency of the energy, the power density of the RF field that strikes the body and the polarization of the wave. Besides, RF energy is absorbed more efficiently thus lead to maximum heating when operating at the frequencies near the body's natural resonant frequency [2]. For example, the adult humans head resonates around $400 \mathrm{MHz}$ and baby's smaller head resonates near $700 \mathrm{MHz}$, thus increasing the RF energy being absorbed as shown in figure 1 . This research was conducted by Profesor Om Ghandi one of the scientist in University of Utah in 1996.

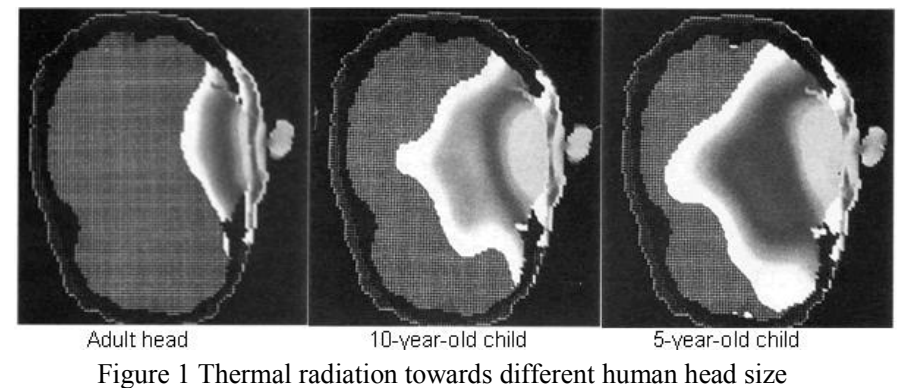

The levels of energy absorbed is measured using a specific term called the specific absorption rate (SAR) and expressed in watts per unit mass of tissue, $\mathrm{Wkg}^{-1}$. SAR is used for the frequency between $100 \mathrm{kHz}$ to $10 \mathrm{GHz}$ which depend on the geometry part of the body exposed, the location and the source of the energy [3]. SAR is the mass-normalized rate of an electromagnetic energy absorbed by the body at a specific location $(\mathrm{x}, \mathrm{y}, \mathrm{z})$ and represented using the equation [1].

$$
S A R=\frac{\sigma|E|^{2}}{\rho}[W / g]
$$

where, $\sigma$ is tissue conductivity, $\rho$ is tissue mass density and $E$ is the root mean square (RMS) value of the induced interval field strength.

There is a general agreement that the main demonstrable effect on the human body above $100 \mathrm{kHz}$ is thermal effect due to the transfer of electromagnetic field energy to the body [4]. The major parts of humans body is consisting of water 